U.S. Department of Commerce

National Technical Information Service

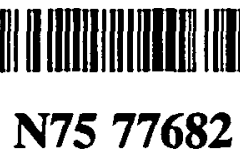

\title{
UNCONVENTIONAL INERTIAL SENSORS
}

\section{ELECTRONICS RESEARCH CENTER}

CAMBRIDGE, MA

1964 
1. AERODYNAMICS, AIRCRAFT

2. AERODYNAMICS, MSSILES AND SPACE VEHICLES

3. AIRCRAFT: all manned atmospheric classes or specific types; components.

4. AIRCRAFT SAFETY AND NOISE

5. ATMOSPHERIC ENTRY: drag devices and forces; reentry maneuvers.

b. ASTRONOMY

7. ASTROPHYSICS

H. HEHAVIORAL STUUIES: psychology; personnel selection and training; human enginer ring.

9. BIOME:DICINE

10. BIOCHEMISTR Y

i1. BIOLOGY

1?. CHEMICAL ENGINEER ING

13. CHEMISTRY, INORGANIC.

14. CHEMISTRY, ORGANIC

15. C.HEMISTRY, PHYSICAL

16. COSMOCHEMISTRY: chemistry of planetary and celestial bodies; and interstellar space.

17. COMMUNICATIONS AND SENSING EQUIPMENT, FLIGHT: satellite instrument payloads

18. COMMUNICATIONS AND TRACKING INSTALLATIONS, GROUND

19. ELECTRONICS

20. FLUID MECHANICS: aerodynamics (except aerodynamics, aircraft and aerodynamics, missiles and space vehicles); hydrodynamics; magnetic-fluid.dynamics.

21. GEOPHYSICS AND GEODESY: meteorology.

22. GUIDANCE AND HOMING SYSTEMS

23. LAUNCHING FACILITIES AND OPERATIONS

24. LAUNCHING DYNAMICS

25. MATERIALS, ENGINEERING: construction materials; properties.

26. MATERLALS, OTHER: lubrication and wear; sealing compounds; hydraulic fluids; coolants; shielding materials; igniters.

27. MATHEMATICS: abstract studies.

28. MISSILES AND SATELLITE CARRIERS: weapons; sounding rockets; satellite launchers.

29. NAVIGATION AND NAVIGATION EQUIPMENT

30. PHYSICS, ATOMIC AND MOLECULAR: structures; spectroscopy; periodic system.

31. PHYSICS, NUCLEAR AND PARTICLE: radiation; nuclear reactions; structures; force fields.
32. PHYSICS, SOLID STATE: cryogenics; crystallography; semiconductors; theories of elasticity, plasticity.

33. PHYSICS, THEORETICAL: classical mechanics other than fluid mechanics; magnetism: optics; acoustics; wave and quantum mechanics.

34. HLOTING: preflight and flight routines: rescue operations.

35. POWER SOURCES, SUPPLEMENTARY: a uxiliary sources; batteries; solar and nuclear generators.

36. PROPELLANTS: characteristics; handling.

37. PROPULSION SYSTEM ELEMENTS: injectors; nozzles; heat exchangers; pumps.

38. PROPULSION SYSTEMS, AIR-JET: turbojets; ramjets; propeller systems. Includes these types using nuclear heat sources.

39. PIOF :ULSION SYSTEMS, LIQUID-FUEL ROCKETS

40. PROPULSION SYSTEMS, SOLID-FUEL ROCKETS

41. PROPULSION SYSTEMS, ELECTRIC: ion jets; plasma jets.

42. FROPULSION SYSTEMS, NUCLEAR: fission or fusion systems using non-ambient working fluids.

43. PROPULSION SYSTEMS, OTHER: systems not assignable to other categories, e. g. solar radiation.

44. PROPULSION SYSTEMS, THEORY: analyses not assignable to listed categories; factors such as combustion parameters, thrust. efficiency.

45. RESEARCH AND DEVEL OPMENT FACILITIES: laboratories; flight ranges.

46. SPACE MECHANICS: orbital calculations and observations.

47. SATELLITES: orbital.

48. SPACE VEHICLES: non-orbital.

49. SIMULATORS AND COMPUTERS: mathematical and physical.

50. STABILITY AND CONTROL: aircraft, missiles, and spacecraft.

51. STRESSES AND LOADS: calculation methods; structural tests; fatigue; vibration and flutter; aeroelasticity; stress analysis.

52. STRUCTURES: design criteria ; component selection.

53. VEHICLE PERFORMANCE: specific flights; observed performance; history.

(Numbers above 53 not assigned.)

$438 \mathrm{r}$

(NASA-TM-X-57626) SENSCES (NASA)
ONCONVENTICNAI INEETIAL

REPRODUCED BY

NATIONAL TECHNICAL

INFORMATION SERVICE

U.S. DEPARTMENT OF COMMERCE DEPARTMENT OF COMMERd
SPRINGFIELD, VA. 22161
Unclas 29513
N 75-77682

$00 / 98 \quad 29513$




\section{$\therefore$ 3}

UNCONVENTIONAL INERTIAL SENSORS

Robert C. Langford Assistant Director for Guidance and Control Research National Aeronautics and Space Administration Electronics Research Center Cambridge, Massachusetts, USA

This paper surveys methods of operation, present state of development, and performance of inertial sensors not using principles of angular (or vibrational) momentum of rigid bodies. Fluid Sphere technology, using a rotating body of fluid, has made possible a gyroscope with two degrees of freedom. Ring Lasers, with their lack of moving parts, have achieved drift rates of a few tenths of a degree per hour. In Electrostatic Gyroscopes, where high voltage breakdown sets a limit to levitation forces, present design projections offer promise of $100 \mathrm{~g}$ operation for small balls. Magnetic Resonance Gyroscopes and Superconductive Gyroscopes are discussed, and an introduction given to newer concepts using Relativistic Sensors, Rotating Tensor Sensors, Vortex Rate Sensors, and Quantum. Mechanical effects:

\section{N.75-77682}
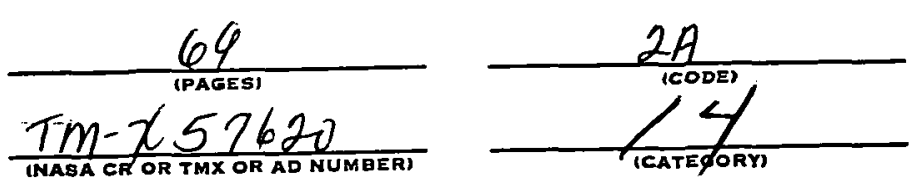
(0) INTRODUCTION

The context of the term Unconventional Sensors used in this paper covers concepts and devices not using angular (or vibrational) momentum of rigid bodies. Such concepts have been regularly examined at conferences such as that sponsored by the Bureau of Naval Weapons, Air Force Systems Command, and Republic Aviation Corporation at Farmingdale, Long Island, New York. Tables $0-1$ and 0-2 give lists of the concepts covered in the unconventional field. Discussion of these concepts has been and indeed, still remains difficult, due to consideration of security and proprietary rights.

After considerable discussion with specialists in many aspects of the field, five devices and four classes of concepts covering phenomenology that may influence future devices emerge as leading candidates for examination. Duncan, in his 1964 State of the Art Review, ${ }^{(0-1,0-2)}$ pointed out that only three laboratory concepts of angular sensors appeared that did not use the concept of angular momentum of spinning rigid bodies:

(a) those using vibratory momentum,

(b) nucleons (which exhibit spin momentum but can hardly be classed.as rigid bodies),

(c) transit time difference of two beams of radiation propagating in opposite directions around the same closed path:

It is the second or concept part of the paper that lists some new ideas that have emerged since. 
(0) REFERENCES

0-1. Duncan, R. C., I.S. Navy, 1964 State of the Art -

Navigation, Guidance and Control, Annual Meeting of the AlA, Washington, D.C., June 1964,

0-2. Duncan, R. C. and Gunnersen, A. A., "Inertial guidance, $\because$ navigation and control systems, Journal of Spacecraft and Rockets, Vol. 1, No. 6, pp. 577-587 (1964). 


\section{(1) RING LASER SENSORS}

The basic principle of operation of the ring laser gyroscope depends on a laser being a light amplifier. Once the working material is in an excited state, it will emit light as a result of the passage of a stimulating beam of light. The emitted light will be found to have the identical frequency, phase, and direction as the stimulating light. One of the requirements for this to happen is that the stimulating light have a frequency which is in one of the several rather narrow bands in which the excited material can emit radiation.

As is true of any amplifier, the laser can become an oscillator if it is coupled to a resonant device and if there is feedback of some of the output energy. The usual laser device consists of a suitable material (solid or gas) placed between two parallel mirror surfaces. The space between the mirrors forms a resonant cavity (resonant at each frequency at which they are separated by an infegral number of half wave lengths) and feedback is inherent in the placement of the lasing material in the cavity. This device will, if the lasing material can be kept in an excited state, oscillate continuously at a frequency which is within the emission band of the material and at which there are an integral number of wavelgngths of light in the round trip between the mirrors. The frequency can be discontinuously changed in these integral jumps over a narrow band by changing the separation of the mirrors.

The ring laser differs from the conventional laser in having three or more mirrors so placed as to cause the light to travel around a closed path (triangular, square or other); the lasing material (presently a He-Ne mixture for $\mathrm{CW}$ operation) is placed in one or more of the legs between mirrors as in Figure 1-1. In principle the ring laser is not different from the parallel mirror form and the possible oscillation frequencies are determined by having an integral number of wavelengths around the entire path. Because of the 
closed nature of the path, however, there can be two distinct light beams involved, one traveling clockwise and the other counterclockwlse. Put another way, there can be two simultaneous ascillations for the two opposed directions of light travel.

For rotation sensing, the pertinent characteristic of the ring laser is that the effective distance around the closed path changes when rotation of the assembly occurs, the length increases for light traveling in the same sense as the rotation while the length decreases for light traveling in the opposite sense. Because of this the resonant frequencies for the cavity change, going up for one direction of light travel and down for the opposite. We thus find that under the influence of rotation the device will have two simultaneous oscillations at different frequencies with the difference being almost exactly proportional to the input angular rate. It should be noted that aside from the effects of rotation, the two paths are identical so that small path variations cancel: To make use of this interesting property, it is necessary to mix samples of the clockwise and counterclockwise beams and apply to a photocell detector. The total light intensity as seen by the cell will vary of the difference frequency and its electrical output will have this same frequency.

Certain of the points which make this an attractive approach to rotation sensing can be stated as follows:

1. The device should, theoretically, be unaffected by acceleration.

2. The device has no moving parts.

3. Its output is inherently digital (frequency is proportional to rate; hence, each count is a small angular increment).

4. The scale factor is a function of a relatively long path which, can be very stable in percentage terms. 
5. Because the speed of light is so great, the effective path length changes are very small for practical rates; for this reason, saturation rate is very high and second order effects are small.

There also are some problems:

1. There are many different cavity oscillation modes possible within the exciting line width and operating conditions must be carefully selected and controlled to secure and maintain the particular mode desired.

2. There is always some coupling between the CW and CCW beams (backscattering from various optical surfaces, for instance), which tends to lock the frequencies together. This effect closely resembles the locking of oscillators in superheterodyning. The result is that the frequencies become distinct only above some effective path length difference or threshold; furthermore, in the region just above this threshold, the frequency difference is a non-linear function of the path length difference as seen in Figure 1-2.

3. There is no sense of rotation direction in the defected signal.

Experience has shown that practical devices can be expected to have a threshold too high to be useful (20 to 100 degrees per hour) due to the frequency locking. The introduction of an offset bias, however, reduces this considerably and additionally has the added virtue of giving rotation sense. A bias can take any form which causes an unbalance in the effective path lengths in the CW and CCW directions; it causes the oscillation frequencies to differ when no input rate is present and pushes the troublesome locking frequency away from zero rate. The bias must, of course, be great enough to place the locking frequency completely out of the range of rates which is of interest. It must be stable enough not to degrade the accuracy of measurement of the 
frequency difference. Bias also permits easy discrimination between $\mathrm{CW}$ and CCW inputs although it is not the only possibility for this.

There are a number of possible forms of bias (including an actual mechanical input), but most promising results have been obtained using a Faraday cell. This cell depends on the fact that the velocity of light in a dense medium is different for light rraveling with a magnetic field than for light traveling counter to the magnetic field as in Figure 1-3. Such a cell can thus be used to create the desired path length unbalance.

Use of a Faraday cell for bias has been found to eliminate the locking problem but it uncovers two new problems. The new problems are bias stability (related to magnetic field strength stability and characteristics of the material used in the cell) and oscillation mode stability. Bias stability is a relatively straightf6rward! engineering problem and is being attacked as such. Oscillation mode stability is a somewhat different kind of problem and requires further discussion.

As has been stated, the ring laser can oscillate at any frequency which is within the narrow bands determined by the width of the exciting line, by characteristics of the lasing material, and in providing an integral number of wavelengths around the closed path. Since there may well be $10^{6}$ to $10^{7}$ wavelengths around the path, it is possible to have oscillation at any of several frequencies as in Figure 1-4. As first order effects, these oscillations are the same for $C N$ and $C C W$ beams and in general cancel; however, this is not true for second order effects. Any random switching of frequencies can be a source of randomness in the output frequency. Because, however; bias is assumed constant, it will be removed as a constant and the net result is a random driff. 
Experience has shown that when using a Faraday bias cell in a moderately well-controlled temperature environment, but with no temperature compensation, the major randomness to be expected is due to the abovediscussed mode changes. Results have been of the order of 5- to 10-degree per hour variations. (These would probably average out to a substantial degree over a long time period, but results of better than one degree per hour would be doubtful.)

The most recent work has been in the direction of frequency locking reduction of the ring by small motions of one of the rings (done piezoelectrically).

There are several possible techniques for a reference against which to do this. The particular approach used by Wing, Macek, and co-workers at Sperry has reduced the randomness in the output to an equivalent rate in the order of one degree per hour. Present indications are that the major source of randomness is now stability (thermal) in the Faraday cell. Studies are being actively pursued in mode enhancement of a single frequency and mode rejection of other frequencies in the cavity that are capable of being excited by the line width.

In terms of size, the ring laser is still relatively large. The recent results have been achieved in a triangular device in a solid homogenous block, sharply reducing scattering and permitting single moding in the cavity.

The status of the ring laser can be summarized as follows:

Present results are approaching conventional gyro performance but are not yet there.

Quantum jumps in performance improvement are still being made. .. Present size is not competitive, but the enclosed area has been 
reduced by a factor of roughly eight in the past year while performance has been improved by a factor of around thirty; thus, it is difficult to predict the future.

Because the output is inherently digital, and because very high input rates can be accommodated, the device appears woll suited for future use in strapdown applications.

Simultaneous attacks on frequency locking and biasing concepts are yielding substontial improvement. 


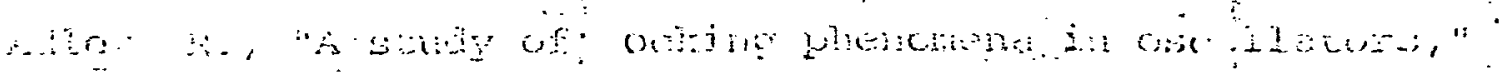

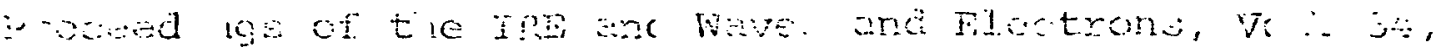

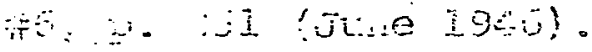

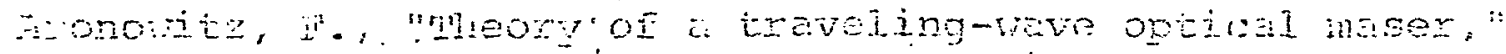

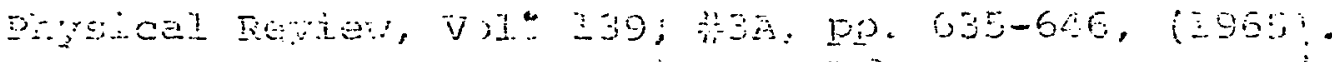

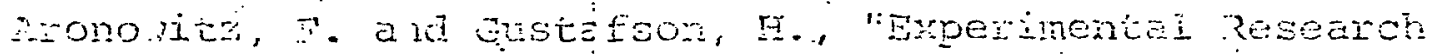

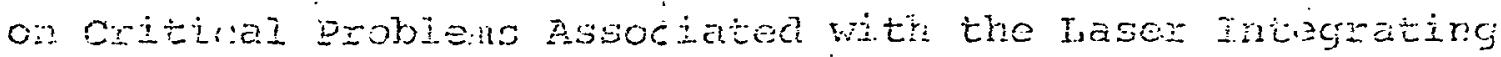
Gyoo," N!. 12018, in 2, Céniract NAS 12-27, (Januaty 1966),

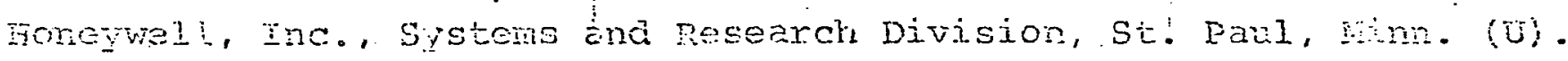

Dagaez, S. I. . Đna oiliers, "spectral characteristics of a gas laser with a traveling wave," JETP Ietcers, Yol. 1, II $(2965)$

Benne t. W. R., Ir., "role burning effects ir a Be-lie : optical naser," The Physiçal Eeview, Vol. 126, \#2, pp. 580-593 $(1962)$

Eershtein; I. I., "Precuercy pulling band of a laser osciliator," DoKlaty, Vol. 10, 607 (1965).

Berschtein, I. H. and zaitsev, Y. I., "Opezation of a gas laser with a ring rescrator." JETP Vol. 49, p.:953 (i965):

Boyd, J. W. and others, "Applied research on iaser Herigation and Guidance, "AFAT-Pr-65-254 (DctoDe- 1965), Systems lesearch Iabs: Inc., Daycon, onio, contract wo. A3 $33(61 ;)-1337 . \because(A D 477.213)(0)$.

collins, S. A. and white. G. R.. "Interferonetric laser moce selsctor," Applied optios, Vol. 2, 44, p. 446! (1963).

gizead, H. L." "Sui les hypotneses relatives a l'eřer

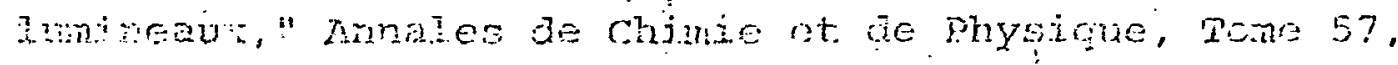
ja.

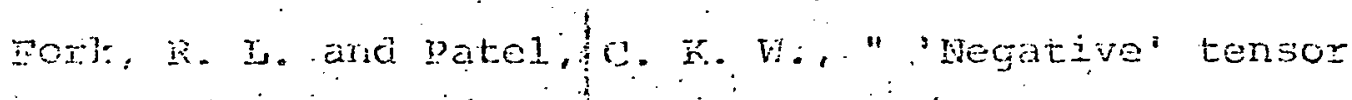

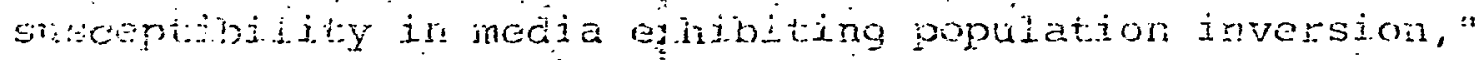

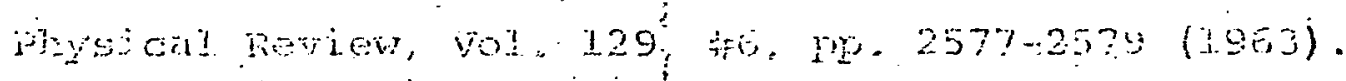




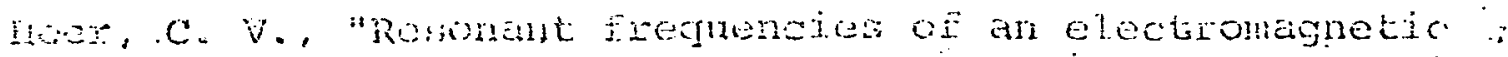

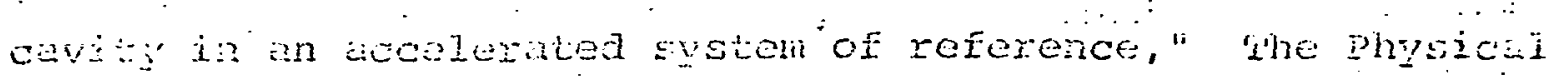
Eevicu, Fol. 134, HA, Pp. 799-E04, (1954).

reen, C. $\bar{v}$ ana matt, R. D., "ritheory of magnetic effecte in optidal maser anplifiers and osciliators," physical Review, VoI. IN0, wa, D. 1030-1:04, (1965).

$\because$ mescher, M. and other, "Ireveling-wave ruby laser with a paseive optical isolatos," Journal of Applied Physics, VOI. 36, 3351 (1965)..

Jenkins, $F$. A. and wite, H. E., Fundamentals of Optics (Mckraw-IIil Boos Co., New york, 1957), 3id ed.

Iandal and Ijifsitiz classified Theory of Fields (Adaison-wesley pubjishing co., Massachusetts, 195i), p. 281.

Iangevin, M. P., "On the theory of reiutivity and the experiment of $M_{0}$ G. Sagnac," Comptes Rendes, vol: i73, p. 831, (1921).

ise,, P.H., "Optical and Mechanical Components for Stabilioed Gas Ring Lasers," Ingineering Report 8093, Contract $A$ 33 (657)-2059, Perikin-Elmer Corp., Electro-opicical Division, Norwalk, conn (October 1965) (AD 472 622)

Letolkhov, V. S. and Marrin, E. P. "On the statistics of laser emission," JETP Vol. 21, p. 509 (1965):

Iittle, $J$. A. and Heer, $C . v$ "Reduction of infrared maser losses by cavity tubing, ". Review of Scientific Instruments:" Vol. $36 ;$; 7 , p. 1061; (1965):

Macek, W. M., and others, "Measurement of fresnel drag with ring lasers," (submitted to Applied physics Letters). Mack: W., and others, Sperry Gyroscope Co., ping Laser Rotation Rate Sensor, Srmposium on Opical Masers, New York, i...., Apri1 j953, proceedings of Symposium (1963), po. 199-207: International Concress on ountum Electronirs. Paris prinoo. Enbruary 1963, Droceddings of Congress (1964), pp. 131:3-1317 (U). 


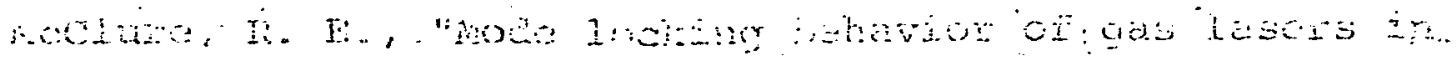

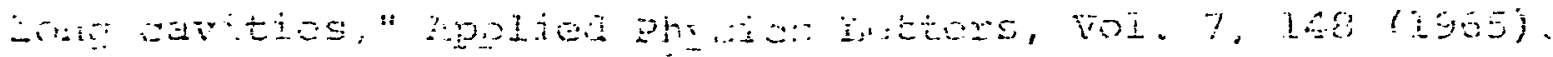

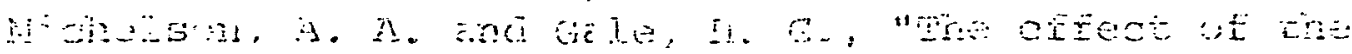

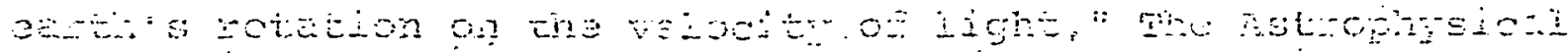
rovend,

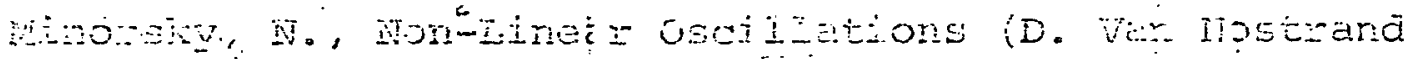

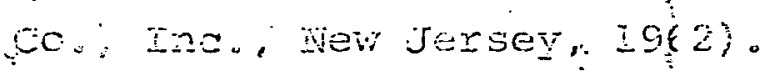

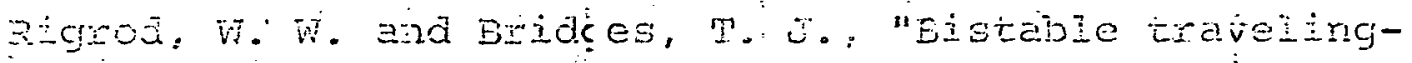

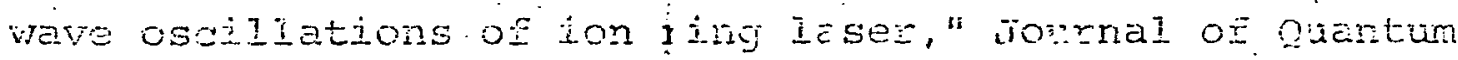
Electronics, Vol. 1, 298 (1965).

"Outpus spectra o: the argon

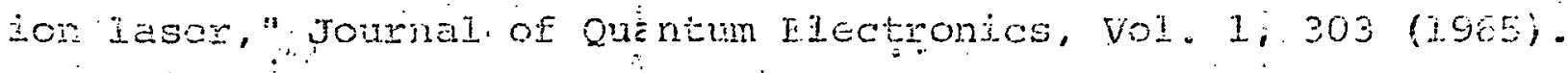
Sagnac, M. G: "Optical turbulating effect - Pho circulation of a Iuminows ether ir a rotating interferoneter," Comptes Rendes, $157,708,(1913) ; J \cdot$ de pinsique, 4 sers, 177. (292I)

Towes, 4 . Electronics (Colvibia unit press, New yorj, is61) p. 3. zeernar; P." "Fresnei's coefficient for light of different colors," Paper 1, nhe Toyel Academy of Amsterdan poceedings (English version), vol. 17, Po. 445-45i (3914). ijod paper 2, vol 18 pe. $398-400$ (1915). 
(2) ELECTROSTATIC GYROSCOPES

The electric vacuum gyro is a two-axis free gyroscope having longterm high precison for inertial navigation. It employs a spherical metal rotor shielded from stray mognetic fields and supported or levitated without physical contact in ultra-high vacuum by servo-controlled high-voltage electric ffelds. The rotor, initially accelerated by induction motor techniques, spins freely with virtually no friction during gyro operation. Fundamental advantages result from the simple geometry, the absence of internal heat dissipation and the elimination of high stress concentrations and wear associated with conventional gyro bearings.

Although the present work has been primarily for nautical applications, the gyro promises high efficiency for spacecraft and satellite applications where low acceleration environmunts permit the use of low voltage, low power consumption rotor support circuitry.

The electric vacuum gyro was invented in 1952 by Dr. Arnold Nordsieck ${ }^{(2-1)}$ while studying the problems of inertial guidance of atomic submarines for accurate launching of Polaris missiles. Realizing that spin-axis bearings were a major source of gyro error, he undertook the study of field-supported rotors. Although magnetic fields easily provide the necessary large forces, troublesome torques result from eddy current losses, hysteresis losses, and rotor magnetic moment. " Theoretical consideration of high-voltage breakdown in vacuum indicated that large forces could also be applied electrostatically without electrical breakdown. Furthermore, lightweight hollow rotors could be supported using the existing state of the art. Since electrostatic forces always act perpendicular to an equipotential surface, torque due to these forces can be minimized by using an accurate spherical metallic rotor for which all forces act through a common center which, by balancing, is made coincident with the mass center. 
Spurious torques, due to electric fields interacting with an inaccurate spherical rotor, constitute the only important limitation to gyro performance.

Stray magnetic fields cause both precession and rotor slowdown torques. The earth's magnetic field, if nat eliminated by shielding, typically results in a slowdown time constant of seven days. To achieve a drift rate of $.0001 \mathrm{deg} /$ hour requires a shielding ratio of about 60 and results by itself in a coasting rundown time constant of 24,000 days. This degree of shielding is readily achieved.

Residual gas causes principally a slowdown torque. The maximum permissible pressure $\left(10^{-5}\right.$ torr) for high voltage breakdown with properly conditioned electrodes results in a rundawn time constant of 15 days. A pressure of $10^{-8}$ or $10^{-9}$ torr, which is rieeded to properly condition the electrodes, accounts for a rundown time constant of 15,000 to 150,000 days. Pressures of $10^{-9}$ torr have been achieved in the gyro, without benefit of bakeout, using a titanium ion pump developed at the laboratory.

Torques can result from a potential gradient along the rotor surface. For this reason, insulating materials, such as an anodized layer which can trap stray charge, must be avoided. Displacement charge flowing over a resistive rotor surface can also produce a gradient resulting in torque. However, this effect is completely' negligible for a clean metallic rotor surface.

Early experimental work employing a hollow spherical rotor which had a "saturn ring" flange for capacitive angle data pickoff was conducted by the Instrument Development and Manufacturing Corporation, ${ }^{(2-2)}$ General Electric Company, ${ }^{(2-3)}$ and Minneapolis-Honeywell Company under Navy contracts. The first tests in 1957 showed large spurious torque 
because of unexpectedly large unbalanced forces acting on the "saturn ring" ' caused by stroy electric fields resulting from unpredictable charge (up to several hundred volts) accumulated on the isolated rotor. At that time, the University of Illinois, Coordinated Science Laboratory, began experimental work using completely spherical rotors, optical dato readout techniques, and a simple resonant circuit, passive element rotor support or levitation serve circuit. (2-5) General Electric and Minneapolis-Honeywell followed with completely spherical rotor gyro designs under both Navy and Air Force contracts. (2-6) Significant improvements in the areas of high vacuum and high voltage vacuum breakdown made at the Coordinated Science Laboratory led to demonstrations of completely reliable electric vacuum gyro operation of both Minneapolis-Honeywell and the University of Illinois in 1959. The encouraging gyro test results stimulated a current Navy requirement for inclusion of electric vacuum gyros as monitors in the existing Ship's Inertial Navigation System (SINS) (2-7). Active electric vacuum gyro development is currently being supported at General Electric, Honeywell, Inc., and the University of Illinois.

Gimballed vs. Gimballess Electric Vacuum Gyro

The torque resulting from the action of electric fields on an imperfectly shaped spherical gyro rotor has led to the development of two basic forms of the electric vacuum gyro.

In the gimballess form, the gyro case is fixed with respect to the vehicle or stable platform and the rotor axis is allowed to assume any direction with respect to the case and levitation electrodes. Optical data readout is obtained by fixed photoelectric microscopes viewing coded patterns which cover a large proportion of the rotor surface and give a measurement of the 
rotor spin-axis angle, relative to the case. This form of gyro is subject to changing torques as changing regions of the imperfectly shaped spherical rotor come under and are acted upon by various electrodes. A compensating computer of considerable complexity is necessary to correct for these torques when highly accurate performance is required.

In the gimballed form of the gyro, the case is mounted in gimbals and servo driven so that the electrodes are always symmetrically located with respect to the rotor spin axis. Since the relative position of rotor and electrodes does not change, torque due to imperfect rotor sphericity interacting with any electrode can be maintained at a null value which is initially achieved by individual fine adjustments of the electrode geometries. The gimbal technique also enables the use of a null-balance type of optical data readout which generally affords greater accuracy. Added complexity is the only apparent disadvantage of the gimballed gyro.

\section{The Gyro Assembly}

The University of Illinois gyro is a gimballed type employing six orthogonally spaced electrodes for levitation, illustrated schematically in Figure 2-1.

The rotor is accurately centered within the electrodes by servo-controlled high voltage. An acceleration of $4 \mathrm{G}$ on a 25 gram rotor is supported by 3800 volts RMS with an electrode gap of $0.025 \mathrm{~cm}$. This gives a field intensity of 150,000 volts per centimeter and a field emission current of 0.5 micro amperes. For a given field intensity, smaller field emission currents result for narrower gaps. Field intensities of 1,000,000 volts/centimeter have been achieved without high voltage breakdown. Two pairs of photoelectric 
microscopes reading a zig-zag pattern around the rotor provide error signals for the gimbal servos which maintain the housing (electrodes and microscopes) in constant alignment with the rotor spin axis. The four induction coils in Figure 2-1 form a poly-phase motor for initial acceleration of the gyro rotor. Present rotors have sphericities of 75-250 nanometer and are balanced to produce a pendulosity period of about three minutes of time . An air bearing support is used for preliminary balancing. Final measurements require the use of an evacuated electrostatic bearing, typically an actual gyro assembly.

Research in high voltage vacuum breakdown has indicated the necessity for clean vacuum systems free of organic vapors. Since precision parts prohibit bakeout of the gyro, vapor pumps are not used. A flask filled with activated charcoal and immersed in liquid nitrogen is used to reduce the system pressure from atmospheric to $10^{-2}$ torr in about one hour. The flask is then valved or sealed off and a Varian Vacion or similar titanium sputtering pump is employed to reduce the pressure to $10^{-7}$ or $10^{-8}$ torr. This pressure is usually maintained for several days before sealing off the gyro. After sealoff, the gyro pressure may rise as high as $10^{-5}$ torr. This drops quickly to $10^{-8}$ torr upon activation of the permanently attached combination titanium ion pump and Bayard-Alpert vacuum gauge. Pressures of $10^{-9}$ torr have been reached in the gyro.

The coils are excited by two phase power at a frequency of 2 or $3 \mathrm{kHz}$. A typical operating speed of 200 revolutions per second is reached in about 15 minutes. Howeveri it takes about a day to dissipate the heat generated in the rotor during runup and achieve stable gyro operation. Figure 2-2 shows also the two Helmholiz coils which are excited by D.C. to damp the 
wobble which results when the rotor is initially accelerated about other than its intendad spin axis and to torque the gyro into initial olionment. A uniform magnetic field creates a torque which tends to bring the rotor spin axis into alignment with the magnetic flux lines and thus the axis of the coils. Angular momentum is lost during forquing and must be made up by additional oparation of the spin motor coils. During gyro operation all coils are de-energized.

\section{Electric Torques}

Simple gyro calculations, for a drift of .0001 degrees per hour using a $5.08 \mathrm{~cm}$ diameter hollow sphere rotating at 500 revolutions per second, show that the line of action of $1 \mathrm{G}$ supporting force must pass within $6.6 \times 10^{-9} \mathrm{~cm}$ of the mass center. Since electric fields act perpendicular to an equipotential surface, a good gyro would inherently result from the use of a rotor so accurate that the centers of curvature for all regions of the surface fall within $6.6 \times 10^{-9} \mathrm{~cm}$ of each other. Since this accuracy is three orders of magnitude beyond realizability, special techniques are employed in the gimballed gyro for nulling the torques due to imperfect rotor sphericity. Centrifugal deformation causes additional elliptical distortion $\left(2.5 \times 10^{-4} \mathrm{~cm}\right)$, which is large compared to machining errors.

For a typical gyro design having $2.5 \times 10^{-4} \mathrm{~cm}$ rotor distortion and drift rate of .0001 degrees per hour, the translational tolerance must be $2.5 \times 10^{-7} \mathrm{~cm}$, which corresponds to a levitation servo capacitance bridge balanice of one part in 100,000. This is a difficult requirement which will probably be relaxed by making use of more spherical rotors specially machined to compensate for centrifugal deformation. 
Status

The gimballed electrically supported gyroscope for Naval use is being successfully operated in the Fleet. Such gyros manufactured by Honeywell have been undergoing tests aboard the USS Compass Island for several years with very gratifying results. Performance specifications have been exceeded. Some results have been limiled by the accuracy of the reference navigation systems. ESG data led to the discovery of otherwise undetected errors in the SINS timing system; i.e., a scalar counting down from the basic 60 cps master time reference would occasionally gain a count because of extranesus noise,

The above gyroscope uses a $7.5 \mathrm{~cm}$ diameter spherical beryllium rotor preshaped to allow for centrifugal deformation. End axis optical readout ("D" pattern) for gimbal followup is employed. The rotor speed decays during operation with a several hundred day $\mathrm{V} / \mathrm{e}$ time constant. Rotor speed, since it determines sphericity, is used in computer compensation of the gyro. This gyro is designed for only $2 \mathrm{G}$ and has a relatively high ratio of rotor-mass to electrode-area.

Although the specification life is exceeded, ultimate burnout of the filament in the combination vacuum gauge and titanium getter ion pump results in vacuum failure typically after 5,000 hours.

Since the existing conventional SINS systems are providing the required performance, ESG's are not currently being installed in new submarines.

A miniaturized version of the gimballed ESG developed for the Air Force by Honeywell has been läboratory-tested.

NASA is interested in a non-gimballed gyro capable of $100 \mathrm{G}$ (2-9)

High voltage breakdown research indicates that with present materials, -whisker growth (and ultimate breakdown) takes place at electric fields $(2-10)$ 
greater than $0.5 \frac{\text { megavolts }}{\mathrm{cm}}$. This provides a levitation force of 11,000 newtons/sq. meter of area and would accommodate $100 \mathrm{G}$ using an as yet undeveloped $5 \mathrm{~cm}$ diamefer rotor which weighs less than 11 grams.

The problems of low drift in a non-gimballed gyro subject to acceleration forces are many. However, short periods of acceleration followed by longer drift-free periods of zero $\bar{g}$ space flightare feasible; and NASA is supporting the development of all-attitude, non-gimballed gyros with two-scale (high $\mathrm{g}$ and very low $\mathrm{g}$ ) levitation systems. General Motors hit Research Laboratories under the direction of Arnold Nordsieck has developed and tested a novel all-attitude readout scheme using a repetitive pattern which covers a large portion of the rotor surface. They also have developed a technique for accurately figuring the spherical surface while the rotor is levitated and spinning at operating speed in vacuum.

There naturally is interest in a combined ESG and electrostatic accelerometer. Honeywell proposes nuclear magnetic resonance as a technique for accurately converting rectified $A C$ levitation current of an existing gyro into frequency for use by the guidance computer. $(2-11)$ Bell Aerosystems has developed an electrostatic accelerometer using variable frequency quantized voltage pulses for the input axis levitation. 


\section{(2) REFERENCES}

2-1. Nordsieck, A., "Feasibility of a Free Gyro Navigation System," Contract No. DA 36-039-SC-56695 (1954), U. of Illinois, Control Systems Laboratory, Urbana, Illinois:

2-2. Instrument Development and Manufacturing Corporation, Contraci No. Nonr 1579(00), Status Reports, September 1956 to June 1957.

2.3 General Electric Company, Contract NObs 72163, Interim Development Report for Free Gyro (Electrostatic), October 1956; Second Inierim Development Report for Electrostatic Gyro, January 1957 ? $\therefore$ (Report MP-77), Future Program--

Electrostatic Gyro Development, October 1957.

2.4 Minneapolis-Honêywell Regulator Company, Contract NObs 72164, Electrostatic Gyro, MH Aero Reports 48221-PR 1-6, (October 1956-January 1958); MH Aero Reports 1502-PR 7-9, (May 1958-December 1958); MH Aero Report 1502-TR 1, (March 1959).

2.5 University of Illinois; Control Systems Laboratory, Contract No. DA 36-039-SC-56695, Progress: Reports, June, July, August, 1957; March, April, May, 1959.

Coordinated Science Laboratory, Contract

No. DA 36-039-SC-85122, Progress Reports June, July; August, 1959;

December, 1960; January, February, 1961.

2.6. Minneapolis-Honeywell Regulator Company, Contract No. NObs 77096, Electrostatic Gyro, MH Aero Reports 1515-QR 1,2, (JulyAugust 1959); MH Aero Reports 1901-QR 3, 4, 5 (1515), (November 1959September 1960); MH Aero Report 1901-TR 1, (April 1961).

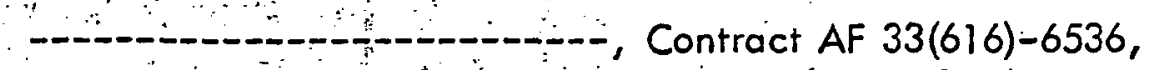

Airborne Electrically Suspended Gyro, Phase 1, MH Aero Report 1517-TR 1, March 1960. 
2.7 Minneopolis-fioneyweli Res. Company, Spery P.O No. 42689, inerial Novigation Monitoring Techniques Study Pragram, MH Aero Report 1905-PR 2, Novemiser 1960; Pririany Design Fucturs Reloted to an For SINS, MH Aero Reporis 190:-TR 1, December 1950; Inertial Navigation Monitoring Techniques Siudy, MH Aero Repo

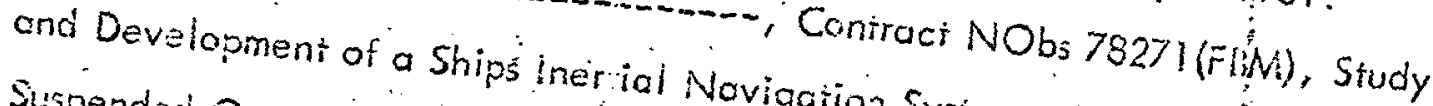
Suspended Gyro, vitt inerial Novigation System using on Electrically 2.8. Minneapolis-Ho i 1900-PR 19, May 1961. Suspended Gyro Unill, Contract NObs 88514 (FBM), Electrically 2.9 Jet Propulsion Lobidy, MD Report 196\%-TR 2, (April to September 1964). on a Strapdown Miniature Electriostatic Gyro-finact No. 95060\%, Study Program No. 1726 FR 2, Phase iB.

Atritude Reference System Utilizing : Contract No. 950915, Study of an 20113 FR I, March 1965. . y an Electrically Suspended Gyro, Report 2.10 Tomaschke, H. E. and others, "Electrical Breakdown in Ultrahigh Vacuum 11, "Journal of Vacuum Science Technology, Vol 1, "2, pp 35-50 (1964). Tomoschike, H.E., "A Study of the Projections of Electrodes and their Effect on Electrical Breakdown in Vacuum," Thesis, U. of Illinois, CSL Repart R-192, January 1964.

2.11 Honeywell, Ine, Aeronautical Div., Feasibility Siudy of a ThreeAxis Electrically Suspended Gyro-Accelerometer with NMR Readout, TR No. AFAL-TR-64-313, February 1955. With NMR Readout, TR No. 
Allen, D. R, "Mininizing precession torques in the:

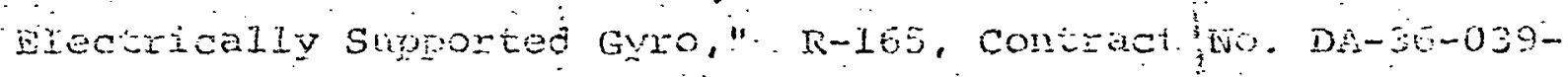
sc-35t22, (May 1953), uo of IIIincis, coordinted seience

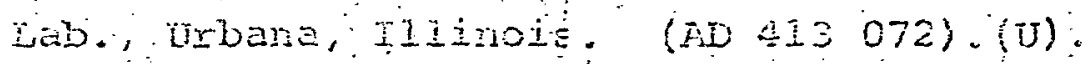

Johrison, $\mathrm{F}, \mathrm{V}$, "Résearch and Development of a Bodybound Irertial Navigation Eystem using Electrostatically Supportéd pendulous Gyro Accelerometers, Contract No. AP $33(616)-3421$ (ApriI 1963), Geileral Eleteric.

Nordsieck, A., Principles of the EVG, ERS Gilidance,

\section{\&.} Control and Navigation. Conference, Stanford untversity. August 1961 .

Mathematical Estimates of Electric Vacuum Gyro Residual Torques" General Motors jefense Research Laboratories Santa Barbara, californta, PR IS6, September 1964

Glaperdas, D. and Inoebel. H. "CSI Electric Vacuun Gyro," Contract No DA-36-039-SC-85122, (Febriary 1964), U: of Iinois, Coordinated Science Lab, Urbana, II jinois. (AD 349496$)=(C)$ 


\section{(3) FLUID SPHERE}

The fluid sphere as pioneered by Sperry is illustrated in its most - elementary form in figure 3-1. It is seen to consist of a rotating member containing a spherical cavity which is completely filled with a liquid. Because of the rotation of the cavity the liquid body is also in rotation (at the come apeed) and possesses angular momentums for this reoson, the liquid body attempts to maintain a coristant spin axis direction in space. The liquid body, being spherical, is free to spin about an axis other than the cavity spin axis; for small space rotations of the direction of the cavity spin axis the fluid body spin axis will, therefore, continue to point its original direction in space.

The above describes a free gyroscope and, in the absence of viscous shear torques, the operation would be completely as described. Because there are viscous shear torques produced whenever there is a rotation of the cavity spin axis away from the direction of the liquid body spin axis, the liquid body spin axis is precessed back into alignment with the cavity spin axis. This is essentially an exponential response and the time constant is given, with reasonable accuracy in a boundary layer derivation by:

$$
\tau=\frac{R}{5} \sqrt{\frac{2}{\Omega \nu}} \quad \text { A tie ticic cosult is } \tau=\frac{R}{0.72} \sqrt{\frac{2}{\Omega v}}
$$

where $R$ is the covity radius in $\mathrm{cm}$.

$\Omega \quad$ is spin rate in radians per second.

$v$ is kinematic viscosity in stokes:

$T$ is time in seconds. 
- The fluid sphere is thus seen to be a rate gyroscope, having the above time constant in its response. It may also be considered a displacement gyroscope in which the displacement angle decays.

The relative shear between the cavity walls and the liquid body can be shown to take place in a relatively thin boundary layer that permits consideration of the liquid body moving as a unit.

Thus the liquid rotor acts much like a solid ball having a diameter slightly smaller than that of the cavity. This ball is separated from the cavity walls by a thin lubricating layer of the liquid. To make use of the gyroscopic properties of this "free rotor" some means must be provided to sense the displacement angle between the spin axes.

Figure 3-1 indicates ports through the cavity wall at points about $45^{\circ}$ removed from the spin axis of the cavity. It will be seen from this figure that; if an angle, $(\delta)$ exists between the spin axes, the ports are at unlike radii with respect to the spin axis of the liquid body; for this reason, the centrifugally induced pressures at the two ports are unequal. One half revolution " after the indicated condition the two ports will have switched positions so that the differential pressure will have reversed sign. An alternating pressure, therefore, exists between the ports, and a differential microphone placed in a connecting passage will sense a pressure varying a spin frequency. The pressure is given by:

$$
P=\rho \Omega^{2} R^{2} \delta \sin (\Omega t+\Phi) \text { dynes } / c \pi^{2}
$$

where $\rho$ is liquid density in grams per cc.

$\Omega \quad$ is spin rate in radians per second

$R$ is cavity radius in $\mathrm{cm}$.

$\delta$ is angular deflection in radians.

is a phase angle and a direct measure of the direction in space of the axis about which the rotation, $\delta$, has occurred. 
It may be seen that the output of the microphone will go to zero when the angle $\delta$ goes to zero, and that a pair of phase sensitive demodulators, using as references the outputs of a two phase alternator coupled to the spin axis, will produce voltages proportional to rotations about two orthogonal axes lying in the plane normal to the cavity spin axis.

The above discussion has indicated, in broad terms, the theory of the gyroscope. Some added detailed statements are in order.

The center of gravity of the liquid ball is inherently coincident with the effective point of support, whether or not the cavity is an accurate sphere; this assumes that the liquid is uniform in temperature. Only axial thermal gradients are important; obtaining good mass balance (low $g$ sensitive drift) is thus, reduced to control of axial thermal gradients.

The liquid body is inherently isoeleastic. Even though the liquid is compressible and some CG shift results from acceleration, this shift is always along the acceleration vector.

The sensitive element is completely non-magnetic so no drift results from close proximity to strong magnetic poles.

Because the spin motor is external, no delicate power leads are required to introduce power. The motor can be large, is easily cooled, and does not dissipate heat in the sensitive element.

The differential pressure transduced by the microphone goes to zero when the displacement angle goes to zero. Because the pressure of interest is alternating it is easily sensed and a static shift of diaphragm position produces no output signal. for this reason 
there is no hysteresis error or time dependent bias error. It should also be noted that the null of the alternating pressure coincides with the zero displacement between the spin axes even if the sensing ports are at unequal angles with respect to the cavity spin axis.

Because the spin bearings are external to the sensitive element, position shifts in these bearings are not important. Furthermore, they can be large and well lubricated; this leads to long life and permits bearing selection to withstand high accelerations.

Because the density of the liquid is of secondary importance, accurate temperature control is not required. Variations in temperature do affect the viscosity of the liquid and, hence, the scale value; even. this is relatively small, however, because the viscosity appears under the radical and because a low temperature viscosity index liquid can be used (about lpercent per degree F).

Pressure transducers can be used in pairs, as shown in Figure 3-2. Used in this way external vibration effects tend to cancel. In addition, the diaphragms can be made to have reasonably the same density as the liquid, thereby almost totally eliminating sensitivity to external vibration.

The optimum spin speed is low, leading to good life and rapid reaction.

Only simple moderate tolerance parts are required and no special assembly or adjustments are needed, so the construction costs are very low. 
- The device is inherently two degrees of freedom and it is only necessary to demodulate the signal in demodulators using as their references the outputs of a two phase alternator coupled to the gyroscope shaft to obtain the two signal components.

The status of the fluid sphere gyro work at Sperry as of March 1965 can be summarized as follows:

A design for a practical, usable device exists. See Figure 3-3. This gyro is about $11.5 \mathrm{~cm}$ long, $6 \mathrm{~cm}$ in diameter and weighs about $0.9 \mathrm{~kg}$.

Results of fests show fluid sphere:

Acceleration sensitive drift $<1^{\circ} /$ hour $/ g$.

Random drift $.1^{\circ}$ per hour.

Bias < $1^{\circ}$ /hour.

Has been subjected to $30 \mathrm{~g}$ centrifuge test. Tests after centrifuging showed no change of $g$ sensitivity, bias or random drift.

Has been subjected to vibration from 10 to $3000 \mathrm{hz}(10 \mathrm{~g}$ above $56 \mathrm{hz}$ ).

No anomolous behavior during vibration, no change of characteristics after.

Has been subjected to $35 \mathrm{~g}$ shock test, no change of characteristics.

Work is continuing toward further refinement with present - emphasis on low cost tactical missile guidance applications. Unit is less than half the previous size. 


\section{(O) REFERENCES}

Dizmond, fi. B., Flud lobr Nutation Sensor for Setellite Venicies," i2th Annuel East Cosst Conterence on Aerospace and Navigational Electronics, Estitrore, Maryland, October 19ós, to be published by Western Periodicals Co., N. Holiywood, Calif.

Vakhaviic, N. N.: "Stability of spin angular velocity of gyroscope with a fluid filled cavity," Prikladnaia Matematika i Mekranika, Vol 29, pp. 35-45, (19ó5) 


\section{(4) MAGNETIC RESONANCE GYROSCOPES}

Magnetic resonance gyroscopes refer to the class of gyroscopic devices which obtain their space reference information from a collection of atomic or nuclear magnetic moments. This type of gyro is also called a nuclear gyroscope. The basic principles of operation have been reviewed and various approaches to their instrumentation described. ${ }^{(4-1)}$ Within the past year there has been an increase in the interest and activities in this area as shown by the new approaches thot have been suggested. Each of the approaches makes use of the common sensing element which has no moving parts and thus eliminates problems directly associated with the manufacture, suspension, and powering of the rotating mechanical mass in a conventional gyroscope : The objectives of the various approaches include development of low cost gyros of modest accuracy, of gyros capable of function in very high g environment, of gyros with high reliability and very long life, and of gyros with extremely high sensitivity.

All of the approaches which have been suggested can be described starting from the equation of motion for a net nuclear magnetization $\vec{M}$ in a magnetic field $\vec{H}$

$$
d \vec{M} / d t=-\gamma \vec{H} \times \vec{M}
$$

where $\gamma$ is the gyromagnetic ratio ${ }^{(4-2)}$. The net magnetization $\vec{M}$ may result from the thermal equilibrium distribution of the nuclear magnetic dipole moments or from some non-equilibrium distribution that results from a process such as optical orientation. In Equation (4-1) all relaxation terms have been ignored. In practical instrumentation of an NMR gyro, these relaxation effects play an important role in determining the limits of operation and 
sensitivity. In a rotating frame of reference, the usual transformation gives

$$
\left.\frac{d M}{d t}\right)_{\text {rot }}=-(\gamma \vec{H}+\vec{\omega}) \times \vec{M} \text {, where } \vec{\omega}_{\text {is angular velocity }}
$$

From observations in $\vec{M}$ and $\vec{H}$, the angular velocity $\vec{w}$ or its time integral may be obtained.

\section{Discussion of Approaches}

The induction gyro in Figure 4-1 is a rate gyro with a two axis response. The device as first conceived detects $\vec{\omega}$ as an equivalent field in a direction perpendicular to the $\vec{H}$ which is composed of two colinear fields $H_{\theta}$. a dc field, and $H_{1}$, an oscillatory field of peak value the same order of magnitude as $\mathrm{H}_{0}$ and angular frequency $\omega_{\mathrm{RF}}=\gamma \mathrm{H}_{0}$. When rotation takes place, i.e. $w \neq 0$, the exact alignment of the dc and ac fields no longer exists. The nuclear magnetization is then caused to precess. The nuclear signal is received in pickup coils tuned to $2 \mathrm{H}_{0}$ and oriented to detect transverse oscillation of the magnetization. The nuclear sample is a proton water $s$ mple doped with paramagnetic ions. It has also been pointed out that the RF drive frequency may be a subharmonic of $\gamma_{0}$ and the fundamental frequency $\mathrm{\gamma H}_{0}$ picked up. ${ }^{(4-3)}$ Several models have been constructed to test various aspects of the theory. Rotation rates of the order of 100 RPM have been detected. A more advanced model of the induction. . gyro has been designed and is currently under construction. This model will be shielded against the earth's magnetic fields. The output noise level will be equivalent to an angular velocity of the order of several 
earth-rate units. The object of this program is a unit with moderately low drift rates and modest cost. In addition to this approach, newer concepts are being explored which employ maser operation. In this instrumentation, two nuclei are also used to eliminate problems associated with magnetic field fluctuations! $(4-4)$

The optically pumped NMR gyro in Figure 4-2 is a device which obtains rotation information from simultaneous observation of steady state NMR signals from $\mathrm{Hg}^{199}$ and $\mathrm{Hg}^{201}$. The rotation is detected as a phase shift between the two signals which are the outputs of an NMR controlled oscillator. This instrument is a single degree of freedom, rate integrating gyroscope, which is well suited for stropdown opplication due to its inherent insensitivity to rotation about any axis except the sensitive axis defined by the magnetic

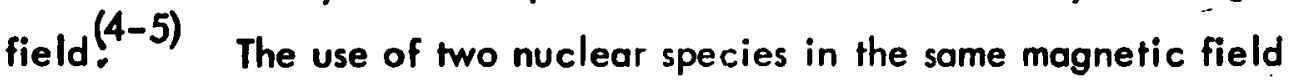
eliminates the need for exact control or knowledge of the magnetic field. Referring to the basic NMR gyro equation (4-2), the $\vec{H}$ field has two components: $H_{0}$ a dc field of moderate homogeneity, and $H_{1}$, an oscillatory field at the frequency $\mathrm{\gamma H}_{0}$, directed orthogonal to $\mathrm{H}_{0}$. The rotation w which is sensed is that component parallel to $H_{0}$. Several models have been constructed and tested. A rather complete theory has been developed.

Experimental operation and theory are in agreement.

Typical test operation of the experimental gyro model of the gyro in laboratory has shown an uncertainty in the drift rate of $0.03^{\circ} / \mathrm{hr}$. These were the results of static runs in which the uncertainty is taken as the rms deviation of the slope as calculated by least mean square methods? 
Recent developments in methods for producing regions with dimishingly small magnetic fields have now made appear possible a nuclear gyroscope in which the net nuclear magnetization is simply isolated from its surroundings. Referring again to the fundamental equation (4-2), when the magnetic field $H$ is made equal, to zero, any apparent motion or change in direction of the net nuclear moment would be determined only by the rotation of the observation apparatus in inertial space.

An experiment has been proposed to test the Lense Thirring effect through observation on an $\mathrm{He}^{3}$ gyro in a satellite ${ }^{(4-7)}$ This is further referenced in Section 6. The general theory of relativity predicts a small perturbation on the motion of satellites about an astronomical body which is rotating. ${ }^{(4-8)}$ A gyro carried in polar orbit would yield the greatest effect. The long NMR relaxation times observed in liquid $\mathrm{He}^{3}$ would give a gyro with sufficiently long life and sensitivity to test the existence of this effect.

Each of the proposed approaches to the instrumentation of an NMR gyroscope has required development of experimental techniques for solution of problems posed essentially by the approach taken. One problem common to all approaches to construction of an NMR gyro is that of magnetic field stability. In fact rotation and magnetic field have the same apparent effect on the dynamics of the nuclear magnetization; this necessitates the control or knowledge of the magnetic field to the equivalent of the desired error in measurement of rate of rotation in most approaches. In the induction gyro, this is done by means of shielding against stray fields and control of current in the coils which generate the field. In the optically pumped NMR gyro, $\therefore$ the question of field control is answered through the use of two kinds of 


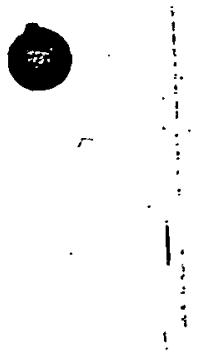

nuclei in the sample. In this case, the magnetic field is treated as an unknown equivalent to the rotation and eliminated through the appropriate instrumentation for this solution of the equations. In the $\mathrm{He}^{3}$-gyro, the problem of the magnetic field control is to be solved by reducing the magnitude of the field to such a small value that any fluctuations in its value will not influence the accuracy of the measurements.

\section{Conclusion}

The feasibility of constructing instruments of useful sensitivity has been demonstrated by using experimental laboratory techniques. Several models of gyros have been operated under laboratory conditions. Future efforts will be concerned with the development of practical instrumentation of the proven techniques and to search for new materials and methods.

In order to see what sensitivities such devices may have, Culver has extended his analysis of the theory of NMR gyros to those in which the nuclei must be reoriented once every relaxation time ${ }^{(4-9)}$ Using presently observed relaxation times, an ultimate sensitivity of better than $.001 \% \mathrm{hr}$ is indicated. 


\section{(i) REFERENCES}

4-1. Simpson, J. H., J., "Nuclear gyroscopes," Astronjutics and Aeronourics, Vol. 2, "̈10, pp. 42-48 (1964).

4-2. Abragam, A.., Principles of Nuelear Magnetism (Oxford Univ. Press, New York, 1961), p. 19.

4-3. Hanuise, G:, Office National D'Etudes et de Recheirches Aerospatiales, Resultats sur le Cyrometre A Induction Nucleaire, Les Gyroscope Avances, Paris, France, Novermber 1964, Published in Proceedings (1955), • pp. 525-534.. (U).

4-4. Minneman, M. J and others, Républic Aviation Cotporation, Recherches sur les Gyroscopes Ä Resonance Magnetique Nucieaire, Les Gyroscopes Avances, Paris, France, November 1964, Published in Proceedings (1965), pp. 505-524. (U):

4-5. Simpson, J. H., J. . "Experimental Model of Nuclear Gyro," (AD 431-727), Contract No. AF 33(6́16)8329, (1964), General Precision, Inc., Aerospace Group, Pleasantville; N. Y. (U).

4-6. Simpson, J. H., Jr., General Precision, Inc., Recent Developments in Optically Pumped NMR Gyroscope Study, Collogue sur le Gyroscopes Avances, Pciris, France, November 1964, (U).

4-7. Fairbank, W. M. "Near Zero: A Frontier of Physics," Twentyfourth Richtmyer Lecture of American Association of Physics Teache $5_{\text {" }}$ Annuai Meeting of American Physical Socieiy, New York, N.Y., (1965):

4-8. Moller, $C_{\therefore}$, Theory of Relativity (Oxford Univ. Press, New York, 1952) p. 317

4-9. Culver, W:, Paper presented ar Les Gyroscopes Avances, Paris, France, November 1964 (paper not available). 
Polonsky, N. and Cohen-Tennoudïi, C., "Pompage optique transveral dans un champ magnefique moduie en amplitude," C. R. Acad Sci, Paris 260, 5231 (1965).

Simpson, J.H., J. "Fi.st quarterly repori on research and cevelopment program on Optically Pumped Nuclear Gyrcscope," GPL-A-33-1, Contract

$\because$ NOw-65-0577-d, (1965), General Precision; Inc., Aerospace Group, Little Falls, New Jersey.

program on Optically Pumped Nuclear Gyroscope," GPL-A-33-2, (1966). "Zimmerman, J.E. and Mercereau, J.E., "Compton wavelength of superconduçing electrons," Physical Review Letters, Vol. 14, 887 (1965). 


\section{(5) SUPERCONDUCTIVE GYROSCOPES}

The superconducting ayro has offered promise of obtaining excellent constancy since il operates at a temperature approaching absolute zero where materials are chemically inactive, expansion coefficients approach zero and creep tendency of materials is reduced. Superconducting bearings are used to suspend the gyro rotor on a thin film of magnetic flux which permils high speed rotation in vocuum using a bearing which is free of wear and friction. The rotor is accelerated to operating speed with a superconducting motor which is practically lossless. Rotor alignment is maintained during spin-up by superconducting coils of a torquer assembly: During grro operation these coils are used to apply error compensating torques. Such devices offer great hope of using effectively the intrinsic characteristics of outer space, lie., zero " $\mathrm{g}$ " and low temperature.

The diamagnetic property of a superconductor is used to obtain bearing action and to produce rotational torques to the rotor. Magnetic flux lines do not penetrate the superconductor and are compelled to flow paralle! to its surface, resulting in a force perpendicular to this surface which is proportional to the second power of the flux density. Somewhat unexpectedly, small hysteresis losses occurred in superconductors when a varying field was impressed on the surface even though the critical magnetic field was not exceeded. Such surface losses were found to be produced In the rotating rotor during every revolution due to changes in flux density resulting from occeleration forces. Since the rotor operofes it a high vocuum these losses must be minimized to avoid ovetheating
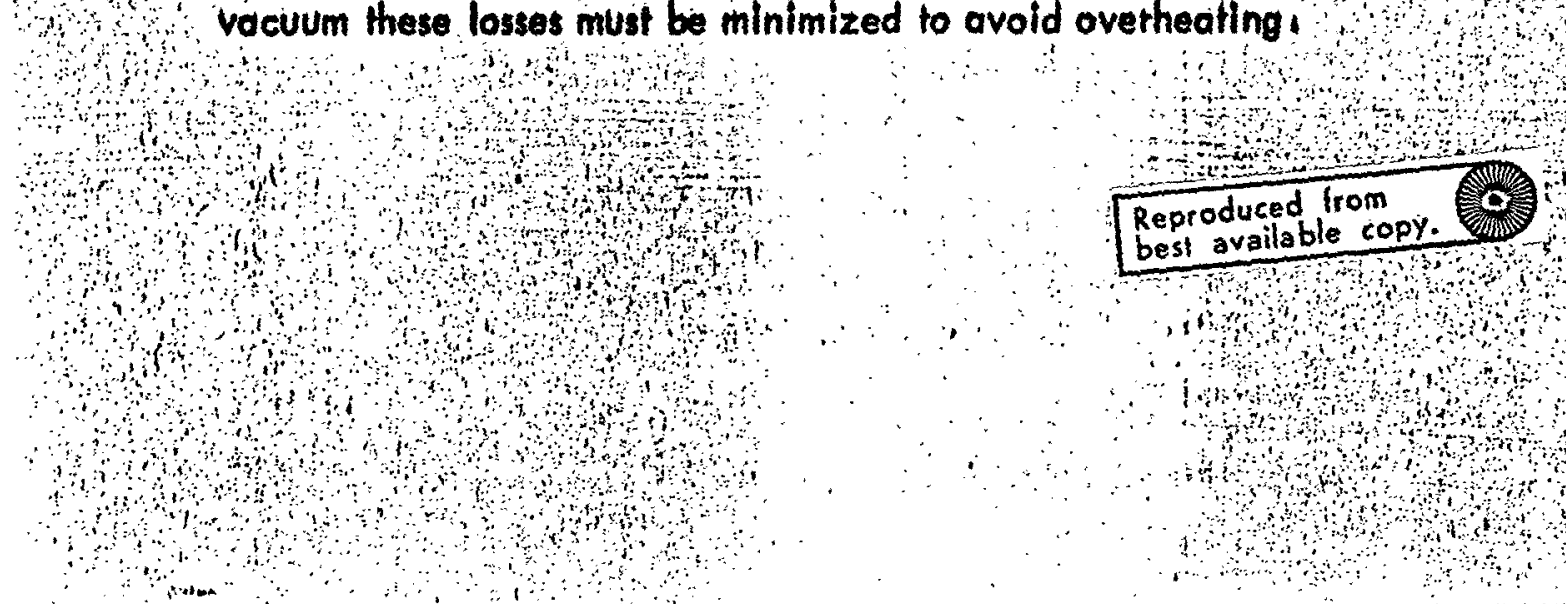
Reproduced from
best available copy.
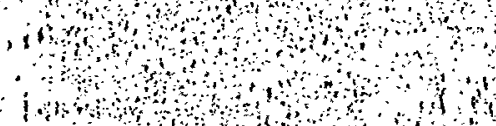

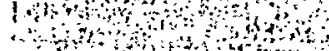

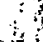


In earlier work, Harding ${ }^{(5-1)}$ reported the rather compelling evidence of their existence when niobium spheres absorbed enough energy whilst rotating in the magnetic field to cause the thermally isolated rotors to exceed this superconductive critical temperature in a matter of minutes and thus lose support. Critical magnetic field and surface losses are influenced by impurities, surface strain, geometry and other factors. Figure 5-1 shows typical loss properties of vacuumrannealed niobium measured with a-c magnetic fields at a frequency of $290 \mathrm{hz}$.

The tendency of niobium to trap magnetic flux requires that the gyro be shielded from ambient magnetic fields during cooldown. In addition it is important that the critical magnetic field not be exceeded during gyro operation. Superconductors such as niobium-zirconium and niobium-tin, which remain superconducfing at extremely high fields, have the property of permitting flux penetration at low density values and are therefore not useful for the gyro bearing.

Harding ${ }^{(5-2)}$ has published an analytical solution to the problem of support field configuration leading to simple but useful coil systems shown in Figure 5-2. These coils carrying opposing currents create a constant gradient field.

$$
\text { B } \propto \text { gradient } r^{2}\left(3 \cos ^{2} \varphi-1\right)
$$

in spherical coordinates which gives stability in all dimensions. By adjusting the strength of the gradient it has been found possible to eliminate magnetic torque in a rapidly spinning ellipsoidally deformed rotor with respect to degree of eccentricity or orientation of its axes relative to the coils. 
The application of the superconducting bearing to support the spherical gyro rotor in an early General Electric model gyro is shown in Figure 5-3. Two bearing coils cause a magnetic flux to flow into the clearance gap around the rotor formed by the superconducting surfaces of the bearing assembly. This flux is required to flow through a fixed gap near the equator which is provided to improve bearing stiffness. Both vertical and lateral support is provided by these bearing coils which are wound around the outside of an evacuated cylindrical housing in which the gyro is mounted.

A superconductive motor used to spin the gyro rotor to a speed of $12,000 \mathrm{rpm}$ is located within a thin non-magnetic enclosure which permits the rotor to operate in vacuum while the stator windings are immersed in liquid helium. Since a rotating magnetic field will not spin a smooth superconducting cylinder, a series of elongated slots is provided in the center of the rotor against which the rotating field can react. As illustrated in Figure 5-4 stator flux which enters the slots is distorted along one edge of the superconducting wall and results in a force which produces a torque. A two-phase stator winding is used to produce a rotating flux needed to spin the rotor. The motor is energized with frequency control obtained from an optical pickoff which views a slotted mirror machined in the rotor surface. Rotor synchronism is thereby automatically maintained until operating speed is reached and the motor de-energized. The time constant of rotor speed decay has been measured to be five months and should increase as rotor balance and surface properties are improved.

Torquers are used to maintain rotor erection during acceleration to operating speed and to provide error torque compensation during operation. Two torquer :- 
assemblies are located in the center of the rotor; one above and one below the motor, each containing four small superconducting coils interconnected to produce torques about two orthogonal axes normal to the spin axis. An optical pickoff used in an early gyro model was located outside of the cryogenic environment of the cryostat to permit use of conventional optical components. The rotor was viewed through a port provided with several vacuum-sealed thermal radiation absorbing windows.

In General Electric's current unit of the gimballed cryogenic gyro, major improvements relate to the bearing configuration and to the use of an optical readout system in which all parts operate at liquid helium temperature. Design features of this new gyro are shown in Figure 5-5. The bearing permits separate control of rotor stiffness along each of three orthogonal axes to give improved " $g$ " capability and better accuracy, since the rotor will be maintained closer to the geometric center of the bearing than was possible in the earlier version.

The new cryogenic optical system follows recent development of small high efficiency light-emitting diodes which, together with suitable semiconducting sensors, have permitted design of an extremely sensitive spin axis position sensing system using a reflecting flat surface formed on the rotor, tilted approximately one-half degree from the rotational axis. This results in the reflected image moving in a conical path which passes through a circular aperture to o germanium sensor. The amount of light received by the sensor varies with the misalignment of the rotor spin axis and the optical axis. With perfect alignment a constant amount of light passes through the aperture and 
no a-c signal output results. As the rotor tilts, an a-c signal is produced which has a magnitude proportional to the tilt angle. Information obtained from a rotor position reference slot used as part of the rotor optical system is used to obtain phase relationship of the spin axis signal to determine direction of rotor tilt.

Considerable progress under AF and NASA sponsorship by Harding, Buchhold, Schock, etc., has been made on gimballed and a bodybound version of this type of gyro. A gimballed cryogenic gyro has shown a constancy of drift at least as low as 0.005 degrees per hour during several test runs using different rotors.

Throughout development of superconducting gyroscopes it has been necessary to conduct special investigations of superconducting properties in order to define design limits and to evaluate the effects of manufacturing processes upon these characteristics.

Future improvement in gyro performance is seen by the use of niobiumcoated ceramic rotors rather than the all-niobium rotors now available. The ceramic rotor will have higher dimensional stability and be lighter in weight. Acceleration force capability up to approximately twenty-five " $g$ 's" is predicted with the ceramic rotor. Excellent superconducting properties have already been obtained with niobium coatings on ceramic.

The gimballed cryogenic gyro has been shown to offer high accuracy potential, and no fundamental barriers to eventual future application have. been uncovered. As advances are made in cryogenic technology, the low. temperature environment becomes less of a challenge. Growing interest and research in the metallurgy of superconductors also will contribute to this program. 


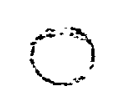

\section{(5) REFERENCES}

5-1. Harding, J. T., J 3 Propulsion Laboratory, Drift Data for the Cryogenic Gyroscope (U), Symposium on Unconventional inertial Sensors, Farmingdale, N.Y., 1964, publ. in Proceedings of Symposium (May 1965), pp. 299-309. (S).

5-2. - . "Force and torque on a superconducting, ellipsoid in an axially symmetric magnetic field, "JPL TR No. 34-242, February 1961. - JPL Research Summary No. 36-5, 1, p 20.
$-\ldots-1$, JPL Fesearch Summary No. 36-8, p. 11. spherical superconductor in an axially symmetric magnetic field." JPL Space Programs Summary No. 37-24, Vol. IV, p. 35, December 1963.

Buchhold, T.A., "The magnetic forces on superconductors and their applications for magnetic bearings,". Cryogenics, Vol. I, \#4, pp. 203-211, (1961). - Applications of superconductivity," Scientific Americgn, Vol 202, p. 74, (1960).

_..._. Generol Electric, Superconductive Gyros, Gyrodynamics Symposium, Celerina, Switzerland, August 1962, Publ. Kreiselproblema (1963).

Buchhold, T. A. and Molenda, P. J. . "Surface electrical losses of superconductors in low frequency fields, Cryogenics, Vol 2, \#6, pp. 344-347 (1962).

Harding, J. T. and Tuffios, R. H., "Cryogenic gyros levitated by magnetic repulsion," Space/Aeronautics, Vol. 36, "3, pp. 133-141 (1961). Schock, K.R., "Superconducting cryogenic motors," Annual Review in Advances in Cryogenic Engineering, Vol . 6, (1960). 


\section{(6) RELATIVISTIC SENSORS}

At Stanford University a program to produce a special purpose gyro of unusual accuracy has led to some contribution to the state of the art. The special purpose is to check certain aspects of the General Theory of Relativity; $^{(6-1)}$ and the requisite drift rate is $.01 \mathrm{sec} /$ year.

Hope for achieving the low drift is based first on operation in a zero $g$ satellite, $(6-2,6-3)$ which will track the spherical gyro rotor without touching it, thus eliminating the ne ed for rotor support. The satellite will also shield the rotor environmentally; thus, it is hoped, drift-producing moments will be lower by $10^{10}$ than for an earth-bound gyro. At this point three contributions are reported.

1. To test the satellite translation control system, a two-dimensional translation-motion simulator--anair.cushionvehicle riding over a granite gauge block surface $(1.2 \mathrm{~m}$ by $1.5 \mathrm{~m})$ is now in operation at Stanford. This facility is the translational analogy of the rotary air bearing tables used for simulation of space vehicle attitude control. The granite block is servo-leveled to a few tenths of an arc second, and the friction level of the $10^{-2} \mathrm{~cm}$ air film corresponds to less than $10^{-7} \mathrm{~g}$. Deliberate tilt of the table corresponds directly to aerodynamic drag in orbit, and the table angle can be programmed to simulate drag variation due to orbit ellipticity, atmospheric non-uniformity, etc. Operation at 200 nautical miles altitude is now being simulated with accuracy.

2. A major problem in conducting the relativity experiment.will be readout of the gyro spin vector--particularly since the spherical rotor must be nearly iso-inertial (to one part in $10^{6}$ ) to reduce gravity-gradient torque to 
a level compatible with the desired drift rate. To solve this problem Professor Fairbank, of the Stanford Physics Department, has invented a technique of possible general interest. Exploiting his own discovery that magnetic flux in a superconductor is quantized, he will use a superconducting unmarked iso-inertial spherical rotor, and sense the London magnetic moment, which is aligned precisely with the spin axis. A special readout circuit (also superconducting) will automatically give a digital readout in flux quanta corresponding to .001 arc sec. The London moment has been detected with the special circuit in Professor Fairbank's laboratory using a very small cylindrical test rotor.

3. An "active damping" technique ${ }^{(6-4)}$ for forcing a chosen axis of a free-rotor spherical gyro to align itself with the gyro spin axis automatically during spinup is being developed by Professor Lange. A rotor, iso-inertial to one part in $10^{6}$, can be aligned in the same amount of time that it requires to accomplish spinup, while conventional passive damping could require months. This technique is useful because any rotor-fixed readout method, such as an optical flat for the unsuipported gyroscope or rotor markings for an EVG, ; requires that the rotor spii, about a known preferred axis. Air bearing laboratory models are being tested.

Principal investigators for the program are Professor Cannon, Professor Fairbank, and Professor Lange. Project Manager is Dr. D. B. DeBra. 
(6) REFERENCES

6-1. Schiff, L. 1., "On experimental tests of the general theory of relativity." Journal of Physics 23, p. 340 (1960).

6-2. Cannon, R. H., Stanford U., Requirements and Design for a Special Gyro for Measuring General Relativity Effects, Gyrodynamics Symposium, Celerina, Switzerland, August 1962, Publ. Kreiselproblema (1963), pp. 146-160.

6.3 Longe, B., "The drag-iree satellite," AlA Journal 2, 9, pp. 1590-1606 (1954).

6-4. Longe, B., Stanford U., The Unsupported Gyroscope (U), Symposium - on Unconventional Inertial Sensors, Farmingdale, N.Y., 1964, publ. in Proceedings of Symposium (May 1965), pp. 157-178 (S). 


\section{(7) ROTATING TENSOR SENSORS}

It has been widely assumed that it is impossible to distinguish between gravitational and inertial effects. (Einstein's Principle of Equivalence) This is true for a point; however, for a finite sized body, Roberson, Diesel, and Forward have found that these effects possess different detectable tensor differences.

Inertial Fields

The linear acceleration, Figure 7-1, of a mass creates a uniform inertial field in the frame of reference of the vehicle which has purely vector properties and no spatial gradients. If the mass is rotating, Figure 7-2, the rotation sets up a symmetrically cylindrical inertial field. This not only has a radial gradient resulting from the change in the magnitude of the acceleration vector with a change in radius, but also a tangential gradient due to the change in direction of the acceleration vector with a change in angle. The resultant acceleration gradient field is a tensor of value zero in the direction of the rotation axis and value $\Omega^{2}$ in the directions at right angles to the rotation axis. The gradient is constant and has no higher order gradients.

\section{Gravitational Fields}

The gravitational force field is equivalent to a gravitational acceleration field

$$
q_{k}=\frac{1}{m} \nabla_{k} \approx-\frac{G, j}{R^{2}}
$$


This can be detected, provided that the center of mass of the sensor and the object being sensed are not in free fall. If the object is in free fall with respect to the sensor the only measurable components of the gravitational field are the grovitational force gradients which comprise a symmetric tensor:

$$
\Gamma_{i i}=\nabla_{i} \nabla_{i} \frac{\varphi}{m}: \frac{G M}{R^{3}}
$$

The spatial pattern of the gravitational force gradient fields can be seen in a simplified form in Figure 7-3.

If a differential force sensor or gradiometer is rotated through this spatial pattern, the output of the sensor will go through a maximum twice each revolution, while any residual unbalanced acceleration forces will cause excitation of the sensor only once per revolution. This double frequency effect and the more general properties of tensors can be used to separate the effects of acceleration, rotation and gravitation by frequency filtering techniques.

In Diesel's gravitational gradient detector, see Figure 7-4, an accelerometer mounted to a freely rototing double bob pendulum will not sense disturbance of the pivot axis since angular acceleration of the pendulum exactly concel the component along the accelerometer sensitive axis. The gravity gradient signal is not similarly cancelled since the mass of the bob is. concentrated as two points $90^{\circ}$ apart in space, and thus $180^{\circ}$ apart with respect to the double frequency sinusoidal signal. 
Forward's gravitational mass sensor, Figure 7-5, consists of a mass spring system with one or more vibrational modes which is rotated at some subharmonic of the vibrational mode. The presence of a non-uniform gravitational field excites the vibrational modes of the structure at twice the rotational frequency.

The readout of the very small vibrations $\left(2.510^{-11}\right.$ to $\left.2.510^{-14} \mathrm{~m}.\right)$ is achieved by the use of piezoelectric strain transducers mounted on the sensor arms. These transducers have been used in previous work to measure ac motions down to $2.5 \times 10^{-16} \mathrm{~m}$.

\section{Present Status}

Forward's cruciform mass sensor is under experimental investigation on NASA Contract NASw-1035. The sensor, when stationary, has already detected ac gravitational nradient signals as small as $10^{-8} \sec ^{-2}$-or about one percent of the earth's gradient. The major effort at present is the reduction of noise introduced during rotation. The noise level in the rotating sensor at present is about 15 times the signal expected from the earth's gradient and is due to turbulence in the vacuum chamber at 0.010 Torr as the sensor "windmills" through the residual air. A better vacuum system and a co-rotating vacuum chamber are being added to eliminate this source. of noise. 


\section{(7) REFERENCES}

Carroll, J. 1. and Suvet, P. M., "Space navigation and exploration Jy gravity difference deiection, All AErospace Engineering $18 \mathrm{pp} .47-47$ (1959).

Crowley, J. C., and otters, "Soire properties of the graviantional field cnd their possible application to space nayigation," IRE Transactions SET 4, pp. 47-54 (i959).

Diesel, J. W., "A new cpproach to gravitational gradient derermination of the vertical," AIAA Journal ?, pi. 1189-1196 (1964).

Forward, R. L., "Rotating tensor sensors," Eulletin of American Physical Society 9, p. 711 (19ó4).

Forward, R. L., Hughes Research Labs., Gravitational Mass Sensors, Symposium on Unconventional Inertial Sensors, Farmingdale, N.Y :, Nov. 1963, published in Proceedings of Symposium, pp. 3ó-60, AD 354-334 (S).

Forward, R. L., Hughes Research Labs., Rotating Gravitational and ineritial Sensors, AlAA Unmanned Spacecraft Nieeting, Los Angeles, Calif., March 1965.

Forward, R. L., Measurement of Static Force Field Gradients, U.S. Patent Application No. 372,866, filed June 1964.

Roberson, R. E., "Gravity gradient determination of the veritical," . ARS Journal 31, pp. 1509-1515 (1961).

Roberson, R. E., U.C.L.A., Establishment of the Center of Mass and Rotational State oi a Space Vehicle by Inerital Techniques, XV inténational Astronautics Congress," Warsaw, Poland, Sepiember 1964, iniern. Aero. Absis. A65-10855, (January 1965).

Savet, P., "Attitude control of orbiting satellires at high eccentricity," ARS Journal 31, pp. 1577-1582 (1962).

Streicher, M., and others, An Inerital Guidance Technique Usable in Free Fall, Natl. Aero. Elect. Conf., Dayton, Ohio, 1959, pubished in Proceedings of Conference, pp.768-772. 


\section{(8) VORTEX RATE SENSOR}

Primary interest in this device is due more to the fact that the output of the vortex sensor may be used directly with fluid amplifier type elements, rather than using the sensor to obtain superior performance over conventional gyroscopic devices. This, no doubt, accounts for the fact that development of the vortex sensor is being performed by workers active in fluid systems work rather than gyro instrument engineers.

\section{Simplified Operating Principles}

The exact solution of the three dimensional equations of motion for a real, viscous, compressible fluid (Novier-Stokes equations) is not known except for a limited number of cases. Normally, a number of simplifying assumptions are made, which reduce the complexity of these equations. In the case of the vortex sensor, it is possible to make a sufficient number of these assumptions to permit a basic understanding of the unit.

Figure 8-1 shows the general configuration of the "pancake area" in the vortex rate sensor. Fluid (liquid or gas) is supplied under pressure to a plenum chamber surrounding the coupling element. This coupling element is made from porous material and will impart any angular velocity $(w)$ of the sensor to the fluid passing through. If it is assumed that the flow is two dimensional and that the fluid is inviscid and incompressible, the following analysis can be made.

If the sensor is rotating with an angular velocity $(w)$ the tangential velocity of the fluid of the coupling element $\left(V_{0}\right)$ is given by

$$
V_{0}=\omega R_{0}
$$


From the conservation of angular momentum, the tangential velocity of the fluid at any radius $(R)$ is inversely proportional to the radius, or

$$
V R=K
$$

If $V_{i}$ is the tangential velocity of the fluid at the sink, then

$$
V_{1} R_{1}=V_{0} R_{0}
$$

or

$$
V_{i}=\frac{V_{0} R_{0}}{R_{i}}=\frac{\omega R_{0}^{2}}{R_{i}}
$$

It can be seen from Equation $(\beta-4)$ that the vortex flow, in effect, amplifies the tangential velocity imparted to the fluid at the coupling element.

The velocity in the radial direction $(U)$ can be determined by the conservation of mass,

$$
\mathrm{UA}=\mathbf{K}
$$

or

$2 \pi R_{0} h U_{0}=K$

where $h$ is the height of the vortex chamber and $U_{0}$ is the radial velocity of the fluid at the coupling element. If the chamber is of constant height

$$
2 \pi R_{i} h U_{i}=2 \pi R_{0} h U_{0}
$$


From this relationship, it is seen that the radial velocity increases as the fluld approaches the sink. This also presents a practical limitation which determines the minimum value that the radius of the sink may approach. The simple one dimensional, isentropic flow equation gives the following relation between velocity and area

$$
\frac{d A}{A}=\left(1-M^{2}\right)\left(-\frac{d U}{U}\right)
$$

where $M$ is the Mach number of the fluid. The velocity, therefore, in the converging passoge cannot exceed the velocity of sound. A limit is established on the sensitivity of the vortex unit, which is now a function of Ro/Ri and Uo, the entrance velocity of the fluid.

Another significant parameter in the vortex rate sensor is the transport delay. Since rate information is generated at the coupling element and is sensed in the sink region, there is a delay associated in the output with: respect to the input. The delay is equal to the time required for a fluid particle to travel from the coupling element to the sink. The ideal transfer function 'can be expressed as

$$
\text { Output }=K \text { (input) } e^{-\tau s}
$$

where $\tau$ is the transport delay of the sensor. From Eq. $(8-5), i$ it is seen that the radial velocity $(U)$ is inversely proportional to the radius for a constant height vortex chamber, i.e..,

$$
\text { U } \quad \therefore=\frac{K}{R}
$$


1.

The differential equation relating distance and time is:
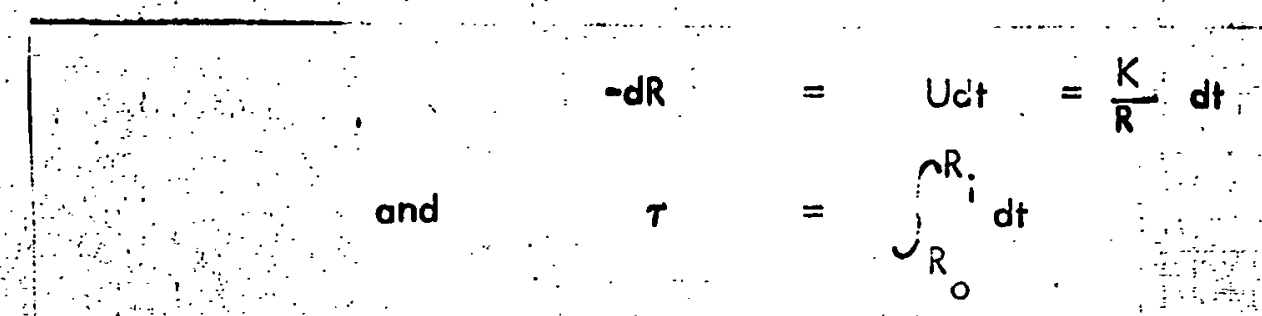

from $E q \cdot 8-11 \cdot d t=-\frac{R c i R}{k}$

and

$$
\tau=\frac{1}{K}\left(\frac{R_{0}^{2}-R_{i}^{2}}{2}\right)
$$

Therefore

$$
\tau=\frac{R_{0}^{2}-R_{i}^{2}}{2 U_{0}^{2}{ }_{0}}
$$


It is evident from this equation that large values of $R_{0}$ and small values of $U_{0}$ which ore required for high sensitivity will also produce large transport delays, degrading the dynamic performance of the unit.

\section{Practical Considerations}

The simplified analysis provides a basic understanding of the vortex rate sensor; however, as with most sensor elements, the performance depends on the sensitivity of the pickoff device. In this case, the fluid rotational velocity at the sink must be sensed in some manner and used to drive a fluid amplifier : In general, however, the relative merits of pickoffs can be measured with respect to sensitivity, inherent noise generation and output power. The weighting factors associated, with each characteristic are a function of the application; therefore, no order of rank may be given.

Noise generation in the coupling element can also present a problem. Although the amplitude of the noise may be small, it will be amplified by the sensor in the same manner as the signal.

\section{Present Status}

The feasibility of the vortex rate sensor has already been demonstrated in providing rate damping signals in an aircraft control system, and has also been used in conjunction with a fluid integrator to provide an attitude reference on an experimental missile control system. In both cases, test flights were highly satisfactory.

The operation of the sensor has been demonstrated on both liquids and gases. The operation of the sensor with sea water as the fluid medium offers a 
wide potential for naval applications. Ram air or water can provide free power in many cases. Compensation techniques to reduce supply pressure and temperature sensitivity are proving successful, and should permit the vortex sensor to operate under environments prohibitive to conventional sensors. The sensor can be made insensitive to radiation, thus could be used on a nuclear-powered vehicle with no shielding. The low mass of the fluid minimizes acceleration sensitivity, making the vortex sensor an ideal instrument for high "G" environments.

The vortex rate sensor is rather unique among the "unconventional" sensors in that its eventual use will be determined primarily on the extent of the application of "fluid amplifier technology." Although operational experience is limited at this time, development tests have been encouraging. The dynamic performance of units presently under development covers control frequencies of $\mathbf{5 h z}$ or less. The vortex sensor offers significant advantages over conventional gyros on the scores of cost and ruggedness; indeed, a unit flown in a test missile was recovered after impact in perfect operational condition, even though the conventional gyro used for telemetry was destroyed.

The ultimate performance potential of the vortex rate sensor has yet to be achieved. There is room for improvement in both the sensitivity and dynamic response of the sensor. Considering the limited research and development effort expended on vortex sensors, the progress to date has been gratifying. However, os the technology receives wider attention, new concepts offering significant improvements will come forth. 
(8) REFERENCES

Fiebig, M., "On the Motion within Fluid Gyroscopes, "AD 608703 , Cont. Af49(638)1262, (1964), Aeronautical Research Associatés of Princeton, Princeton, New Jersèy. (U).

Shapiro, A. H., The Dynamics and Thermodynamics of Compressible Fluid Flow (Ronald Press, New York, 1953-54) Vol. 1. 


\section{(9) QUANTUM MECHANICS}

It is perhaps no more than an intuitive feeling of the author that the best accomplishments in the unconventional sensors will come from examination through quantum mechanics and solid state theory of the fundamental properties of material structure. This type of approach probably optimizes the search for microminiaturized "electromechanical" type sensors compatible with electronic microcircuity. This last section lists some of the very attractive ideas being sturied by Jacobs and his co-workers at Autonetics.

\section{Helicon Gyro}

The helicon gyro concept can be described as a circularly polarized electromagnetic wave that propagates with extremely slow phase velocity along the magnetic field lines of a plasma. Helicon wave frequencies as low as a fraction of a cycle per second have been detected in a small sample of sodium. Since the frequency of the circularly polarized waves should be determined by the physical properties of the plasma alone, the measurement in inertial space of this frequency would be independent of the state of rotation of the solid. If, however, the measurements were made in a rotating frame of reference, the frequency would be shifted by the rotation rate. Hence, rotation rate might be measured as a shift in helicon frequency. Because helicon frequencies are low, and frequencies can be measured with great precision, a sensitive rate sensor should result. By choosing two helicon waves in two plasmas with opposite fields, the vehicle rotation could be produced as a direct output of the sensor. Such a device has no moving mechanical parts, and has the virtues of simple construction, and small volume. It would have no $g$ sensitivity and, hence, have utility as a high $g$ and rate device. 


\section{Diode Laser Accelerometer}

An interesting acceleration sensing device has been designed taking advantage of the properties of solid state injection diode lasers. It has been shown at Autonetics that the lasing frequency of GaAs diodes can be tuned by hydrostatic pressure. Preliminary results indicate that the stress sensitivity is such as to yield a very sensitive device with a wide dynamic range. The instrument is mechanized by supporting a proof mass between two diodes which are caused to lase. The optical outputs of the two are combined and heterodyned. Under acceleration, the stress on one diode is increased, and decreased on the other, causing a respective increase and decrease in the optical frequency. By mixing these light beams in a photocell, a beat frequency proportional to acceleration is obtained. This instrument is similar in principle to the vibrating string accelerometer with diode quantum oscillator lasers replacing the vibrating strings.

\section{Ferromagnetic and Solid-State Resonance Effects}

The area of oscillatory effects in solids is one where new approaches may lead to stress-frequency transducers having high reliability and long lifetime. Such sensors could be small in size, and have no moving parts, and operate over a wide temperature range at about room temperature with no requirements for a cryogenic environment. Solid-state oscillatory effects can be conveniently divided into four areas:

1. Radio frequency ferromagnetic resonance in uniaxial magnetic thin films,

2. Magnetoacoustic resonance,

- 3. Current oscillations in semi-conductors,

4. Solid-state plasma. 
Radio frequency ferromagnetic resonance (FMR) in uniaxial magnetic thin films may be applied to acceleration-sensing by utilizing the high magnetoelastic sensitivities of certain uniaxial films and by utilizing the high stress sensitivity of FMR to the angle between an applied field and the anisotropy self-field in the film. In terms of $Q$, the $Q$ of FMR is already rather high, but coupled with the high angular sensitivity, the $Q$ is enhanced by several orders of magnitude. A stress-sensor of high resolution is therefore anticipated.

Magnetoacoustic resonance is a phenomenon observed at microwave frequencies rather than radio frequencies in materials which have both high- $Q$ magnetic modes and acoustic modes which are rather tightly coupled. The frequency range over which the effect has been observed is from 1 to $10 \mathrm{gHz}$. The sample size is about $0.25 \times 10^{-6} \mathrm{~m}$. For pump powers above a threshold of approximately 3 milliwatts both magnetostatic modes and acoustic modes can be excited. The strong effect of the stress-field upon the acoustic frequency will be communicated to the magnetic system through the electron spin-phonon interaction. The effect of stress may then be observed indirectly through its effect on FMR frequency, which can be determined with good precision.

Current oscillation in semiconductors is another area in which application may be made to a stress-sensor. Small size is again possible; the sensing material itself may be a cube $10^{-2} \mathrm{~cm}$ on a side. The high-Q oscillation, which exists after a threshold value of electric field (about $1000 \mathrm{vol} / \mathrm{s} / \mathrm{cm}$ ) is applied across the sample, depends on both sample dimensions and material properties. Both are expected to change sensitively with stress. 


\section{(9) REFEREINCES}

Helicon Gyro

Bowers, R, and others, "Oscillatory galvanomagnetic e:fect ir merallic sodium," Physical keview letiers, Vol. 7, "79 (1961).

Chambers, R. C. and Junes, B. K., "Measurement of th high-field hall effect by an inductive methind," Proceedings of the Royal Socitety, Londign, Vol. A270, "1342 (1962).

Ferromagnetic and Solid-State Resonance Effects

Crowther, T. S., "Angular and magnitude dispersion of thie anistropy in magnetic films," Journal of Applied Physics 34, p. 580 (1963).

Hasiy, T. E. and Eoudreaux, L. J., "Ferromagnetic resonance in thin magnetic films at radio frequencies, Journal of Applied Physics 32, p. 1807 (1.961).

Hasiy, T. E. and Penn, T. C., Texas Instruments, Inc., An ulitrasen̂́sitive angular transducer using ferromagnetic film resonance International Conierence on Non-linear Magnerism, Washington, D.C., April 1963, Proceedings of Conference, $\hat{p}_{0} \cdot 16-7-1$. (C).

Smith, D. O., "Anistropy in permalloy films," Journal of Applied Physics 30, p. 264 (1959).

Vescial, $F .$, "FMR measurements of magnetoelastic constants in evaporated uniaxial permalloy films," Bulletin of American Physical Society, Series 11, Vol. 10, $\frac{\| t}{1}$, p. i 7 (1965).

Woli, I. W. and Crowither, T. S., Magnetoelastic sensitivities in evaporated crid electrcdeposited permalioy films, ": Journal of Applied Physics 34, p: i205 (1963; 


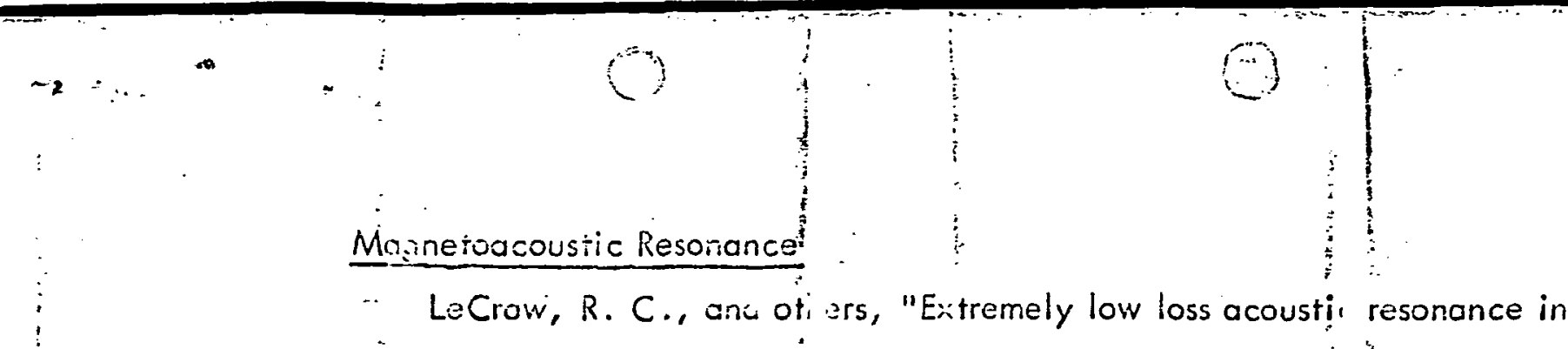
single crystc! garnet spheres," Physical Review Letters 6, p. 620 (1961). Spencer, E. G. and Leïraw, R. C., "Magnetoacoustic resonance in Yitrium iron garnet," Physical Review Letiers 1, p. 241 (1958).

a

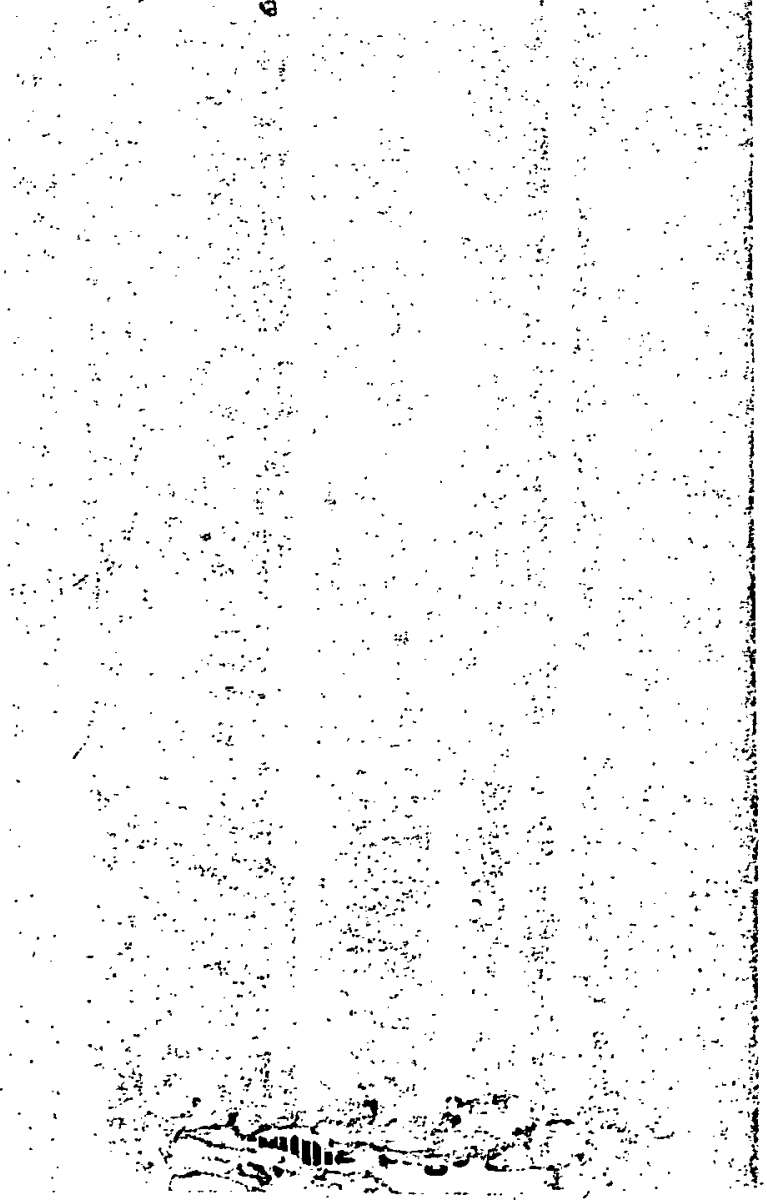




\section{ACKNOWLEDGMENTS}

In a review as wide ranging as this article, authorship in the pure sense tends to depart rather considerably towards editorship. I wish first to acknowledge the encouragement and assistance of Robert H. Cannon, Jr. in writing this article; to Leil Tanenholz in assiduously helping throughout, despite a severe accident halfway through preparation.

Above all, I wish to thank those specialists who contributed so freely of their time and skill in the respective sections: Daniel Debra, Howard K. Knoebel, Willis G. Wing, James H. Simpson, Walter Haeussermann, T. A. Buchhold, K. F. Schock, John T. Harding, Robert E. Roberson, Robert L. Forward, Thomas G. Wetheral, A. E. Schmidlin, Earl D. Jacobs; and the organizers and participants of the annual meeting on Unconventional Sensors, sponsored by Bureau of Naval Weapons, Air Force Systems Command, and Republic Aviation Corporation, Farmingdale, Long Island, N.Y., under the chairmanship of Robert E. Mclntyre.

Finally, I wish to thank my colleagues on the AIAA Guidance and Control Committee for their many suggestions and helpful criticisms. 